Preprint typeset in JHEP style - HYPER VERSION

UB-ECM-PF-05/13

\title{
New Bubble Decays In Kaluza-Klein Theories
}

\author{
Jaume López Carballo \\ Departament ECM, Facultat de Física, Universitat de Barcelona, Spain. \\ E-mail: jlopez@ecm.ub.es \\ Jorge G. Russo \\ Institució Catalana de Recerca i Estudis Avançats (ICREA) \\ Departament ECM, Facultat de Física, Universitat de Barcelona, Spain. \\ E-mail: jrusso@ecm.ub.es
}

\begin{abstract}
We describe new bubble decays in pure $D+1$ dimensional Einstein theory with two compact directions. The instanton solution is constructed by analytic continuation of the Kaluza-Klein electrically charged black hole solution. We show that the instanton describes the decay of a Kaluza-Klein vacuum $\mathbb{M}^{D-1} \times T^{2}$ with a non-vanishing torus tilt parameter. The decay is produced by the creation of a bubble of nothing which expands with time. We compute the instanton action, which shows that this Kaluza-Klein vacuum becomes more stable as the torus tilt parameter is increased. As an application, we consider the decay of M-theory torus compactifications leading to type 0A/0B string theories.
\end{abstract}

Keywords: gravitational instantons, bubbles, Kaluza-Klein. 


\section{Contents}

1. Introduction 1

2. Bubbles decays from Kaluza-Klein charged solutions 2

$2.1 \quad D=5$ black holes with Kaluza-Klein charge 2

2.2 Instanton

2.3 Bubble decay 6

3. The instanton action 7

3.1 Direct calculation

3.2 Computation using thermodynamics 8

3.3 Decay rate 9

4. Decay of Type 0 String Theory 9

A. Decay of $\mathbb{M}^{D-1} \times T^{2}$

A.1 The $(D+1)$-dimensional instanton 11

A.2 The instanton action

\section{Introduction}

Long ago Witten [1] realized that the $\mathbb{M}^{4} \times S^{1}$ vacuum of the original Kaluza-Klein theory is unstable via a semiclassical decay [2, 3] into a bubble of nothing, where a hole forms in space, which rapidly expands to infinity, reaching the speed of light after a short time (we use the notation $\mathbb{M}^{n}$ for Minkowski space in $n$ dimensions).

To construct the bubble solution, Witten starts with the Euclidean version of the $D=5$ Schwarzschild black hole. The Euclidean time, $\tau=i t$, now plays the role of the compact Kaluza-Klein coordinate. The resulting metric represents an instanton solution that interpolates between the false (unstable) vacuum $\mathbb{M}^{4} \times S^{1}$ at $r \gg r_{h}$, and the true vacuum solution, which is obtained by a second Wick rotation, leading to the bubble solution

$$
\mathrm{d} s^{2}=\frac{\mathrm{d} r^{2}}{1-\left(r_{h} / r\right)^{2}}+\left(1-\left(r_{h} / r\right)^{2}\right) \mathrm{d} \tau^{2}-r^{2} \mathrm{~d} \psi^{2}+r^{2} \cosh ^{2} \psi \mathrm{d} \Omega_{2}^{2}
$$

A similar analytic continuation procedure can be applied to construct a different instanton solution starting with the $D=5$ Myers-Perry Kerr solution. By suitable Wick 
rotations, one obtains an Euclidean solution of finite action,

$$
\begin{aligned}
\mathrm{d} s^{2}= & \mathrm{d} \tau^{2}+\sin ^{2} \theta\left(r^{2}-a^{2}\right) \mathrm{d} \varphi^{2}-\frac{\mu}{\rho}\left(\mathrm{d} \tau+a \sin ^{2} \theta \mathrm{d} \varphi\right)^{2} \\
& +\frac{\rho^{2} \mathrm{~d} r^{2}}{r^{2}-a^{2}-\mu}+\rho^{2} \mathrm{~d} \theta^{2}+r^{2} \cos ^{2} \theta \mathrm{d} \chi^{2},
\end{aligned}
$$

where $\rho^{2}=r^{2}-a^{2} \cos ^{2} \theta$. A natural question is then what kind of decay this instanton describes. This question was answered by Dowker et al [四], who showed that the instanton solution has the right asymptotics to describe the decay of Kaluza-Klein vacuum in the presence of a magnetic field, the Kaluza-Klein Melvin background, representing a static, cylindrically symmetric magnetic flux tube. Indeed, at infinity one has a flat metric, which in cylindrical coordinates reads

$$
\mathrm{d} s^{2}=-\mathrm{d} t^{2}+\mathrm{d} z^{2}+\rho^{2} \mathrm{~d} \varphi^{2}+\mathrm{d} \tau^{2} .
$$

Demanding that the metric (1.2) is free of conical singularities at the horizon $r_{h}^{2}=\mu+a^{2}$ leads to the identifications

$$
(\varphi, \tau)=\left(\varphi+2 \pi n R_{\tau} B+2 \pi m, \tau+2 \pi n R_{\tau}\right)
$$

with $n, m \in \mathbb{Z}$ and $B=a / \mu$, which define the Melvin magnetic flux tube. There are two instanton solutions (1.2) that approach the same Melvin magnetic configuration, differing by a shift in the magnetic parameter $B$. They describe two decay modes of this space. One is the instability via nucleation of a pair of monopoles $\left(B=a / \mu+1 / R_{\tau}\right)$, by a magnetic dual of the Schwinger effect of pair creation in an electric field. The second decay mode ( $B=a / \mu)$ is the formation of a bubble of nothing. ${ }^{1}$

Having shown that $D=5$ Schwarzschild solution and the $D=5$ Kerr solution describe instanton decays of Kaluza-Klein vacua, a natural question is whether a similar interpretation holds for the instanton constructed from the Kaluza-Klein electrically charged solution, i.e. a black hole with $U(1)$ charge associated with a Kaluza-Klein gauge field. The advantage of having a charge of Kaluza-Klein origin is that in the higher dimensional theory the instanton is a solution of pure Einstein theory. Here we will show that this instanton describes the semiclassical decay of a Kaluza-Klein space $\mathbb{M}^{4} \times T^{2}$ with a non-zero torus tilt, via the formation of a bubble of nothing. The torus tilt parameter arises as a result of identifications which are necessary to render the space free of conical singularities.

\section{Bubbles decays from Kaluza-Klein charged solutions}

\section{1 $D=5$ black holes with Kaluza-Klein charge}

We consider pure Einstein theory in six dimensions. We are interested in semiclassical decays of the vacuum $\mathbb{M}^{4} \times T^{2}$ (in the appendix, we consider the case $\mathbb{M}^{D-1} \times T^{2}$ ). One vacuum

\footnotetext{
${ }^{1}$ In string theory, there is in addition a tachyon instability originating from a winding mode which appears above some critical magnetic field parameter [5].
} 
decay is represented by the Witten bubble of nothing generalized by including an extra $S^{1}$ dimension. This is constructed by adding to the five dimensional Schwarzschild black hole metric an extra (isometric) direction $y$, where $y$ is a periodic coordinate, $y=y+2 \pi R_{y}$.

To construct more general decays, our starting point is the electrically charged solution given in [6, 7]. The five-dimensional solution is obtained as the Kaluza-Klein reduction of a six-dimensional metric which is a solution of the Einstein equations in six dimensions, i.e. it satisfies $R_{\mu \nu}\left(g_{6}\right)=0 .^{2}$

The six-dimensional metric is given by

$$
\begin{aligned}
\mathrm{d} s_{6}^{2}= & -\frac{1}{1+\alpha / r^{2}}\left[\left(1-\left(r_{h} / r\right)^{2}\right)-\frac{Q^{2}}{8 \pi^{4} r^{4}}\right] \mathrm{d} t^{2}+\frac{\mathrm{d} r^{2}}{1-\left(r_{h} / r\right)^{2}} \\
& +r^{2} \mathrm{~d} \Omega_{3}^{2}+\left(1+\alpha / r^{2}\right) \mathrm{d} y^{2}+\frac{Q}{\sqrt{2} \pi^{2} r^{2}} \mathrm{~d} y \mathrm{~d} t .
\end{aligned}
$$

The coordinate $y$ is periodic with $y=y+2 \pi R_{y}$. The dimensional reduction to five dimensions in $y$ is obtained by writing the metric in the form

$$
\mathrm{d} s_{6}^{2}=\mathrm{e}^{2 \phi / 3} \mathrm{~d} s_{5}^{2}+\mathrm{e}^{-2 \phi}\left(\mathrm{d} y+A_{\mu} \mathrm{d} x^{\mu}\right)^{2} .
$$

One finds that the five dimensional metric describe a black hole with mass $M$ and charge $Q$. Its line element is

$$
\mathrm{d} s_{5}^{2}=-\frac{1-\left(r_{h} / r\right)^{2}}{\left(1+\alpha / r^{2}\right)^{2 / 3}} \mathrm{~d} t^{2}+\frac{\left(1+\alpha / r^{2}\right)^{1 / 3}}{1-\left(r_{h} / r\right)^{2}} \mathrm{~d} r^{2}+\left(1+\alpha / r^{2}\right)^{1 / 3} r^{2} \mathrm{~d} \Omega_{3}^{2} .
$$

The gauge field is given by $A_{\mu}=\left(A_{0}, 0,0,0,0\right)$, where

$$
A_{0}=\frac{Q}{2 \sqrt{2} \pi^{2}} \frac{1}{r^{2}\left(1+\alpha / r^{2}\right)} \text {. }
$$

Finally, the scalar field is

$$
\phi=-\frac{1}{2} \ln \left(1+\alpha / r^{2}\right)
$$

The solution is fully specified by two parameters, namely the mass $M$ and the charge $Q$. Introducing a parameter $k$

$$
k=1-\sqrt{1+\frac{3}{2}\left(\frac{Q}{\kappa^{2} M}\right)^{2}},
$$

the different parameters can then be written in terms of $M$ and $k$ by means of the following relations

$$
\begin{aligned}
Q^{2} & =\frac{2}{3}\left(\kappa^{2} M\right)^{2} k(k-2), \\
r_{h}^{2} & =\frac{\kappa^{2} M}{3 \pi^{2}}(1+k), \\
\alpha & =-\frac{\kappa^{2} M}{2 \pi^{2}} k .
\end{aligned}
$$

\footnotetext{
${ }^{2} \mathrm{~A}$ discussion (which has no overlap with the present treatment) of bubble solutions related to fourdimensional Kaluza-Klein charged black holes is in [B]. Discussions of other Kaluza-Klein bubble solutions can be found in 9,10 .
} 
The solution represents a black hole with regular horizon in the region

$$
0 \leq\left(\frac{Q}{\kappa^{2} M}\right)^{2} \leq 2
$$

This corresponds to the range $-1 \leq k \leq 0$. For larger values of $Q / M$, the metric exhibits a naked singularity.

\subsection{Instanton}

We can obtain an Euclidean solution from solution (2.1) by Wick rotations, $t \rightarrow i \tau$ and $Q \rightarrow i q$. The latter is necessary in order to have a real metric. So the Euclidean solution reads

$$
\begin{aligned}
\mathrm{d} s_{6}^{2}= & \frac{1}{1+\alpha / r^{2}}\left[\left(1-\left(r_{h} / r\right)^{2}\right)+\frac{q^{2}}{8 \pi^{4}} \frac{1}{r^{4}}\right] \mathrm{d} \tau^{2}+\frac{\mathrm{d} r^{2}}{1-\left(r_{h} / r\right)^{2}} \\
& +r^{2} \mathrm{~d} \Omega_{3}^{2}+\left(1+\alpha / r^{2}\right) \mathrm{d} y^{2}-\frac{q}{\sqrt{2} \pi^{2} r^{2}} \mathrm{~d} y \mathrm{~d} \tau .
\end{aligned}
$$

We stress that this is a solution of pure (Euclidean) Einstein theory, where the action contains just the Einstein-Hilbert term,

$$
I=\frac{1}{16 \pi G_{6}} \int \mathrm{d}^{6} x(\sqrt{g} R)_{6},
$$

without any additional field. For $q=0$, the solution reduces to the Witten instanton with an extra $S^{1}$ coordinate $y$.

The geometry has a potential conical singularity at the horizon which must be removed as usual by a suitable identification. The norm of the Killing vector,

$$
\begin{gathered}
\zeta=\partial_{\tau}+A \partial_{y}, \\
A=\frac{q}{2 \sqrt{2} \pi^{2}} \frac{1}{\alpha+r_{h}^{2}},
\end{gathered}
$$

vanishes at the horizon. We introduce a new coordinate $\tilde{y}=y-A \tau$, which is constant along the orbits of $\zeta$. In terms of this coordinate, the metric takes the form

$$
\begin{aligned}
\mathrm{d} s_{6}^{2}= & \frac{1-\left(r_{h} / r\right)^{2}}{1+\alpha / r^{2}} \mathrm{~d} \tau^{2}+\frac{\mathrm{d} r^{2}}{1-\left(r_{h} / r\right)^{2}}+r^{2} \mathrm{~d} \Omega_{3}^{2} \\
& +\left(1+\alpha / r^{2}\right)\left(\mathrm{d} \tilde{y}+\frac{q}{2 \sqrt{2} \pi^{2}}\left(\frac{1}{\alpha+r_{h}^{2}}-\frac{1}{\alpha+r^{2}}\right) \mathrm{d} \tau\right)^{2} .
\end{aligned}
$$

Near the horizon the relevant part of the metric reduces to

$$
\mathrm{d} s^{2} \approx \frac{2\left(r-r_{h}\right)}{r_{h}\left(1+\alpha / r_{h}^{2}\right)} \mathrm{d} \tau^{2}+\frac{r_{h}}{2\left(r-r_{h}\right)} \mathrm{d} r^{2}+\cdots
$$

The horizon is at $r=r_{h}$. In order to avoid the conical singularity in (2.12), $\tau$ must be periodic, with $\tau=\tau+2 \pi R_{\tau}$ at fixed $\tilde{y}$. The periodicity $2 \pi R_{\tau}$ is determined by introducing a Rindler coordinate $\tilde{r}=\sqrt{2 r_{h}} \sqrt{r-r_{h}}$. The relevant part of the metric becomes

$$
\mathrm{d} s^{2} \approx \frac{\tilde{r}^{2}}{r_{h}^{2}+\alpha} \mathrm{d} \tau^{2}+d \tilde{r}^{2}+\cdots,
$$


which shows that the space is free from conical singularity provided $\tau=\tau+2 \pi R_{\tau}$ with

$$
R_{\tau}=\sqrt{r_{h}^{2}+\alpha}
$$

This gives the Hawking temperature $T_{H}=\left(2 \pi R_{\tau}\right)^{-1}$.

At $r=\infty$, the instanton (2.7) approaches the metric

$$
\mathrm{d} s_{6}^{2}=\mathrm{d} y^{2}+\mathrm{d} \tau^{2}+\mathrm{d} r^{2}+r^{2} \mathrm{~d} \Omega_{3}^{2},
$$

with the identification

$$
(y, \tau)=\left(y+2 \pi n R_{y}+2 \pi m A R_{\tau}, \tau+2 \pi m R_{\tau}\right) .
$$

Introducing $2 \pi$-periodic variables $\sigma_{1}, \sigma_{2}$ by $\tilde{y}=R_{y} \sigma_{1}, \tau=R_{\tau} \sigma_{2}$, the asymptotic metric is

$$
\mathrm{d} s_{6}^{2}=R_{y}^{2}\left|\mathrm{~d} \sigma_{1}+\Omega \mathrm{d} \sigma_{2}\right|^{2}+\mathrm{d} r^{2}+r^{2} \mathrm{~d} \Omega_{3}^{2},
$$

with $\Omega$ representing the torus modular parameter

$$
\Omega=a+i \frac{R_{\tau}}{R_{y}}, \quad a=\frac{A R_{\tau}}{R_{y}} .
$$

The behavior of the instanton at infinity tells us which is the original vacuum that decays into the bubble. From (2.17) we see that the instanton approaches the space $\mathbb{R}^{4} \times T^{2}$, where the 2-torus has a modular parameter given by $\Omega$, eq. (2.18). The torus modular parameter is a feature which characterizes the Kaluza-Klein vacuum, modulo $S L(2, \mathbb{Z})$ transformations,

$$
\Omega \rightarrow \frac{a \Omega+b}{c \Omega+d}, \quad a, b, c, d \in \mathbb{Z}, \quad a d-b c=1 .
$$

In particular, $a \rightarrow a+1$ gives an equivalent torus. This property is manifest in the identifications (2.16), since $A \rightarrow A+R_{y} / R_{\tau}$ is absorbed into $n$. We shall restrict the modular parameter $\Omega$ to the fundamental domain $\mathcal{F}$ of $S L(2, \mathbb{Z})$. In particular, this implies the restriction

$$
-\frac{1}{2}<a \leq \frac{1}{2} \quad \text { or } \quad-\frac{R_{y}}{2 R_{\tau}}<A \leq \frac{R_{y}}{2 R_{\tau}} .
$$

It is convenient to write the instanton metric (2.7) in terms of the parameters $R_{\tau}, R_{y}$, $A$ that specify the Kaluza-Klein vacuum. Using eqs. (2.5a), 2.10), (2.14) we find

$$
k=\frac{2 A^{2}}{3+A^{2}} \text {. }
$$

Therefore

$$
\begin{aligned}
q & =2 \sqrt{2} \pi^{2} R_{\tau}^{2} A, \\
r_{h}^{2} & =R_{\tau}^{2}\left(1+A^{2}\right), \\
\alpha & =-R_{\tau}^{2} A^{2} .
\end{aligned}
$$


The instanton metric becomes

$$
\begin{aligned}
\mathrm{d} s_{6}^{2}= & \frac{1-\left(1+A^{2}\right) R_{\tau}^{2} / r^{2}}{1-A^{2} R_{\tau}^{2} / r^{2}} \mathrm{~d} \tau^{2}+\frac{\mathrm{d} r^{2}}{1-\left(1+A^{2}\right) R_{\tau}^{2} / r^{2}}+r^{2} \mathrm{~d} \Omega_{3}^{2} \\
& +\left(1-A^{2} R_{\tau}^{2} / r^{2}\right)\left(\mathrm{d} \tilde{y}+A \frac{1-\left(1+A^{2}\right) R_{\tau}^{2} / r^{2}}{1-A^{2} R_{\tau}^{2} / r^{2}} \mathrm{~d} \tau\right)^{2} .
\end{aligned}
$$

The metric is regular on the full (geodesically complete) space $r^{2} \geq\left(1+A^{2}\right) R_{\tau}^{2}$. Due to fact that the shift $A \rightarrow A+n R_{y} / R_{\tau}$ gives an equivalent Kaluza-Klein vacuum at infinity, there will be an infinite family of instanton solutions obtained by replacing $A \rightarrow A+n R_{y} / R_{\tau}$ in the metric (2.23).

Reducing (2.23) in $\tilde{y}$, i.e. along the orbits of the $\zeta$ Killing vector, we find

$$
\begin{aligned}
\phi= & -\frac{1}{2} \ln \left(1-\left(R_{\tau} A / r\right)^{2}\right), \\
\tilde{A}_{\tau}= & A \frac{1-\left(1+A^{2}\right) R_{\tau}^{2} / r^{2}}{1-A^{2} R_{\tau}^{2} / r^{2}}, \\
\mathrm{~d} s_{5}^{2}= & \frac{1-\left(1+A^{2}\right)\left(R_{\tau} / r\right)^{2}}{\left(1-\left(A R_{\tau} / r\right)^{2}\right)^{2 / 3}} \mathrm{~d} \tau^{2}+\frac{\left(1-\left(A R_{\tau} / r\right)^{2}\right)^{1 / 3}}{1-\left(1+A^{2}\right)\left(R_{\tau} / r\right)^{2}} \mathrm{~d} r^{2} \\
& +\left(1-\left(A R_{\tau} / r\right)^{2}\right)^{1 / 3} r^{2} \mathrm{~d} \Omega_{3}^{2} .
\end{aligned}
$$

From the point of view of the dimensionally reduced theory, the torus tilt is a non-zero gauge potential at infinity,

$$
\tilde{A}_{\tau}(\infty)=A
$$

\subsection{Bubble decay}

In the standard treatment of semiclassical vacuum decay [2, 3], the false vacuum decays into a Lorentzian space that coincides with the instanton (the bounce solution) on a threedimensional surface of zero extrinsic curvature at $t=0$. Any Euclidean solution of finite action that has the same asymptotic as the Kaluza-Klein vacuum and that can be analytically continued to a real Lorentzian metric represents a decay mode of the original space.

The Lorentzian signature metric is obtained by a suitable analytic continuation of our instanton solution (2.7). The new time variable has to preserve the symmetry around the hyperspace of $t=0$, so, as in [1], the $\theta=\pi / 2$ plane can be taken as the $t=0$ surface. We write

$$
\mathrm{d} \Omega_{3}^{2}=\mathrm{d} \theta^{2}+\sin ^{2} \theta \mathrm{d} \Omega_{2}^{2},
$$

and perform a Wick rotation $\theta \rightarrow \pi / 2+i \psi$. This leads to the replacement

$$
\mathrm{d} \Omega_{3}^{2} \rightarrow-\mathrm{d} \psi^{2}+\cosh ^{2} \psi \mathrm{d} \Omega_{2}^{2} .
$$

The resulting six dimensional metric contains the terms (see (2.23))

$$
\mathrm{d} s_{6}^{2}=\frac{\mathrm{d} r^{2}}{1-\left(1+A^{2}\right) R_{\tau}^{2} / r^{2}}-r^{2} \mathrm{~d} \psi^{2}+r^{2} \cosh ^{2} \psi \mathrm{d} \Omega_{2}^{2}+\ldots
$$


Introducing coodinates

$$
\rho=r \cosh \psi, \quad \mathrm{t}=r \sinh \psi,
$$

at large $r$ the metric approaches,

$$
\mathrm{d} s_{6}^{2} \cong-\mathrm{dt}^{2}+\mathrm{d} \rho^{2}+\rho^{2} \mathrm{~d} \Omega_{2}^{2}+\mathrm{d} \tau^{2}+(\mathrm{d} \tilde{y}+A \mathrm{~d} \tau)^{2}
$$

So the space at large $r$ is the usual flat space $\mathbb{M}^{4} \times T^{2}$ with a torus tilt. The full space at time $\mathrm{t}$ is a curved spacetime where the region $\rho^{2}-\mathrm{t}^{2}<r_{h}^{2}$, with $r_{h}^{2}=\left(1+A^{2}\right) R_{\tau}^{2}$, has been removed. $r=r_{h}$ is a frontier of the space time: the wall of the bubble. The space is regular at $r \geq r_{h}$.

The radius of the bubble grows with time as

$$
\rho_{\text {bubble }}(t)=\sqrt{\left(1+A^{2}\right) R_{\tau}^{2}+\mathrm{t}^{2}} .
$$

Note that the size of the bubble at $\mathrm{t}=0$ increases with the torus tilt parameter $A$.

The size of the $\tau$-circle at constant $\tilde{y}$ can be read directly from (2.23)

$$
R_{\tau}^{2}(r)=R_{\tau}^{2}\left(1+A^{2}\right)\left(1-\left(1+A^{2}\right) \frac{R_{\tau}^{2}}{r^{2}}\right)=r_{h}^{2}\left(1-\frac{r_{h}^{2}}{r^{2}}\right) .
$$

Thus the $\tau$-circle smoothly shrinks to zero at the surface of the bubble. At infinity, it is $R_{\tau}(\infty)^{2}=R_{\tau}^{2}\left(1+A^{2}\right)$, as follows also from (2.17) by looking at the size of the $\sigma_{2}$ circle at $\sigma_{1}=$ constant.

The size of $\tilde{y}$ circle is given by

$$
R_{y}(r)=R_{y} \sqrt{1-\left(A R_{\tau} / r\right)^{2}}
$$

with $r^{2}=\rho^{2}-\mathrm{t}^{2}>\left(1+A^{2}\right) R_{\tau}^{2}$, so that $R_{y} / \sqrt{1+A^{2}} \leq R_{y}(r) \leq R_{y}$.

\section{The instanton action}

In the semiclassical approximation, the decay rate can be written as the exponential of minus the instanton action,

$$
\Gamma \propto \mathrm{e}^{-I}
$$

We provide two independent computations of the action. By direct evaluation of the action including boundary terms, and by using thermodynamics.

\subsection{Direct calculation}

In six dimensions, the only field we have is the metric, so the action is given by

$$
I=-\frac{1}{16 \pi G_{6}} \int \mathrm{d}^{6} x(\sqrt{g} R)_{6}-\frac{1}{8 \pi G_{6}} \oint \sqrt{h}\left(K-K_{0}\right)
$$

where $h$ is the induced boundary metric, $K$ the trace of its extrinsic curvature and $K_{0}$ the analog quantity for the space where our metric is embedded in (flat space, in our case). 
Since our instanton is a vacuum solution, $R_{\mu \nu}=0$, the only contribution will come from the boundary term.

If $n^{\mu}$ is a unit normal vector to the boundary, the extrinsic curvature is written as

$$
K_{\mu \nu}=\nabla_{\mu} n_{\nu}-n_{\mu} n^{\sigma} \nabla_{\sigma} n_{\nu} .
$$

If the metric is such that $n^{\mu}$ is radial and only depends on the radial coordinate, one can show that the trace of (3.3) reduces to the logarithmic derivative of the square root of the determinant of the boundary metric with respect to a unit radial vector,

$$
\sqrt{h} K=-\frac{1}{\sqrt{g_{r r}}} \partial_{r} \sqrt{h} .
$$

In our case, the metric induced at the boundary is given by (2.7) with $r \rightarrow \infty$ and constant. We get

$$
\frac{\partial}{\partial r} \sqrt{h}=\sin ^{2} \theta_{1} \sin \theta_{2}\left(3 r^{2}-\frac{1}{2} r_{h}^{2}\right)+O\left(r^{-2}\right) .
$$

The flat space term, $K_{0}$, can be obtained from (3.5) imposing $q=M=0$, i.e. $r_{h}=0$. So we have

$$
\sqrt{h}\left(K-K_{0}\right)=-\frac{1}{2} \sin ^{2} \theta_{1} \sin \theta_{2} r_{h}^{2} .
$$

Thus, the action is simply the integral of (3.6),

$$
I=\frac{1}{8 \pi G_{6}} \pi^{2}\left(2 \pi R_{y}\right)\left(2 \pi R_{\tau}\right) r_{h}^{2} .
$$

Using $T=1 /\left(2 \pi R_{\tau}\right)$ and (2.14) we obtain

$$
I=\left(2 \pi R_{y}\right) \frac{\pi^{2}}{4 G_{6}} r_{h}^{3} \sqrt{1+\alpha / r_{h}^{2}}=\frac{\pi^{2} R_{\tau}^{3}}{4 G_{5}}\left(1+A^{2}\right) .
$$

where we have used $G_{5}=G_{6} /\left(2 \pi R_{y}\right)$.

\subsection{Computation using thermodynamics}

In five dimensions, the instanton metric represents an Euclidean charged black hole, whose thermodynamics is well known. The bulk part of the action (3.2) becomes,

$$
I=-\frac{1}{16 \pi G_{5}} \int \mathrm{d}^{5} x \sqrt{-g_{5}}\left(R_{5}-\frac{4}{3}(\partial \phi)^{2}-\frac{1}{4} \mathrm{e}^{-8 \phi / 3} F_{\mu \nu} F^{\mu \nu}\right) .
$$

The action is the ratio between the free energy $F$ and the temperature $T=\left(2 \pi R_{\tau}\right)^{-1}$. The free energy is

$$
F=M-T S-\Phi_{H} Q,
$$

where $\Phi_{H}$ is electric potential at $r=r_{h}$. We can simplify this equation using the Smarr formula [7],

$$
M=\frac{3}{2} T S+\Phi_{H} Q
$$

Thus the action reads

$$
I=\frac{F}{T}=\frac{1}{2} S .
$$


The entropy of the five dimensional black hole is the area of the horizon over $4 G_{5}$,

$$
S=\frac{2 \pi^{2}}{4 G_{5}} r_{h}^{3} \sqrt{1+\alpha / r_{h}^{2}}=\frac{\pi^{2}}{2 G_{5}} R_{\tau}^{3}\left(1+A^{2}\right),
$$

where we have used $(2.24 \mathrm{~d})$. So the action is

$$
I=\frac{\pi^{2} R_{\tau}^{3}}{4 G_{5}}\left(1+A^{2}\right),
$$

in exact agreement with (3.7).

\subsection{Decay rate}

The action is

$$
I\left(R_{\tau}, R_{y}, A\right)=I_{0}\left(1+A^{2}\right), \quad-\frac{R_{y}}{2 R_{\tau}}<A<\frac{R_{y}}{2 R_{\tau}}
$$

where

$$
I_{0}=\frac{\pi^{3} R_{\tau}^{3} R_{y}}{2 G_{6}}=\frac{\pi R_{\tau}^{2}}{8 G_{4}}, \quad G_{4}=G_{6} /\left(\left(2 \pi R_{\tau}\right)\left(2 \pi R_{y}\right)\right) .
$$

The Witten decay [1] corresponds to $A=0$, so that $I_{\text {Witten }}=I_{0}$. In this case the instanton is obtained by analytic continuation from the $D=5$ Schwarzschild black hole. Remarkably, the instanton action $I$ increases with the torus tilt. This indicates that the effect of the tilt $(2.16)$ is to render the Kaluza-Klein vacuum more stable. Intuitively, the reason is that the size of the bubble at the moment of creation $t=0$ is larger the larger is the tilt, so the cost of producing the bubble is greater.

One can also write down the action for the shifted instantons, $A \rightarrow A-n R_{y} / R_{\tau}$,

$$
I\left(R_{\tau}, R_{y}, A\right)=I_{0}\left(1+\left(A-\frac{n R_{y}}{R_{\tau}}\right)^{2}\right),
$$

We see that in the fundamental domain $-\frac{R_{y}}{2 R_{\tau}}<A<\frac{R_{y}}{2 R_{\tau}}$ the dominant decay mode (i.e. the one with less action) is $n=0$. Other decay modes are exponentially suppressed.

\section{Decay of Type 0 String Theory}

In the presence of fermions, the instanton decay studied here (just as in [1]) is relevant for compactifications where fermions obey antiperiodic boundary conditions in the $\tau$ direction. The reason is that the space described by the instanton metric (topologically $\mathbb{R}^{2} \times S^{1} \times S^{2}$ ) admits a unique spin structure in the $\tau$-circle, since this shrinks to zero at the horizon. There are two spin possible structures on $(\tau, \tilde{y})$, namely $(-,+)$ and $(-,-)$, i.e. in the asymptotic region, fermions must be antiperiodic functions in the $S^{1}$ described by $\tau$.

As an application of the decay rate computed here, we consider M-theory compactified on a two torus, where fermions obey antiperiodic boundary conditions in one of the circles. If we regard $\tau$ as the eleventh dimension, this compactification leads [1] to type 0A/0B theories [12]. More precisely, ten-dimensional type $0 \mathrm{~B}$ is equivalent to M-theory compactified on $T^{2} /\left[(-1)^{F} \times \mathcal{S}\right]$ in the limit of zero torus area, where $F$ is the spacetime 
fermion number and $\mathcal{S}$ is the half shift along the circle $(X \rightarrow X+\pi R)$. The type 0B Ramond-Ramond sector has an untwisted subsector $(R+, R+)$ and a twisted subsector $(R-, R-)$. There are two $\mathrm{RR}$ scalars, one of each sector. The tilt of the torus is related to the expectation value of the RR scalar of the untwisted sector.

For a rectangular torus, the $\mathrm{RR}$ scalar field vanishes. The decay rate in this case was studied in [13]. One assumes that there are six compact dimensions $y^{m}, m=1, \ldots, 6$ besides the 2-torus described by coordinates $\tau$ and $\tilde{y}$. The relevant instantons describing the decay are essentially the Witten instanton [1] and the solution of Dowker et al [4. The relation with magnetic fields arises because string theory with antiperiodic fermions in one direction can be described by a Melvin magnetic flux tube backgound with a special magnetic field [5]. For this critical value of the magnetic field, the dominant decay mode is via bubble formation. For small values of the magnetic field, the decay is dominated by creation of $\mathrm{D} 6 / \mathrm{D} \overline{6}$-brane pairs, with a decay rate equal to the Schwinger rate [13] (see [14, 15] for related discussions). In addition to these non-perturbative instabilities, type 0 A theory at weak coupling $g_{0 A}^{2}=R_{\tau}^{2} / 4 \alpha^{\prime} \ll 1$ has a tachyon instability, which makes the theory highly unstable. In this regime, the perturbative decay rate is of order one in $\alpha^{\prime}$ units.

Now consider the decay in the presence of a torus tilt. Consider first the case of five extra directions $y^{m}$ compactified on a rectangular torus, so that we consider the decay of the vacuum $\mathbb{M}^{4} \times T^{2} \times T^{5}$, and we wish to study the effect of a tilt in $T^{2}$ on the decay rate. The relevant instanton is the one constructed in the preceding sections, by trivially adding five extra directions $y^{m}$. The action can be read from (3.15),

$$
\begin{aligned}
I_{0 \mathrm{~A} / 0 \mathrm{~B}} & =\frac{\pi V_{6} R_{\tau}^{2}}{8 G_{10}}\left(1+A^{2}\right)=\frac{4 \pi V_{6}}{(2 \pi)^{6} \alpha^{\prime 3}}\left(1+a^{2} \frac{R_{y}^{2}}{4 \alpha^{\prime} g_{0 A}^{2}}\right) \\
& =\frac{4 \pi V_{6}}{(2 \pi)^{6} \alpha^{\prime 3}}\left(1+\frac{a^{2}}{4 g_{0 B}^{2}}\right), \quad-\frac{1}{2}<a \leq \frac{1}{2},
\end{aligned}
$$

where $G_{10}=G_{11} /\left(2 \pi R_{\tau}\right), V_{6}=2 \pi R_{y} V_{5}$ and $a$ is the expectation value of the vector component $A_{\tilde{y}}$ (or RR scalar in type 0B). We have used

$$
R_{\tau}^{2}=4 \alpha^{\prime} g_{0 A}^{2}, \quad 16 \pi G_{10}=(2 \pi)^{7} g_{0 A}^{2} \alpha^{\prime 4}, \quad g_{0 B}=\frac{R_{\tau}}{2 R_{y}}, \quad a=\frac{A R_{\tau}}{R_{y}}
$$

Thus the decay rate decreases as the tilt $a$ is increased.

Now consider the case where the five $y^{m}$ coordinates are non-compact. The dominant non-perturbative decay mode is again via the formation of a bubble of nothing. For a generic torus with modular parameter $\Omega$, the relevant instanton is obtained from the appendix by setting $D=10$ (this gives the instanton of maximal symmetry). We thus obtain the following decay rate of type $0 \mathrm{~A} / 0 \mathrm{~B}$ theory:

$$
\Gamma_{0 A / 0 B} \propto e^{-I_{0 A / O B}}, \quad I_{0 A / 0 B}=\frac{7^{6} \pi^{4}}{480 G_{10}} R_{y} R_{\tau}^{7}\left(1+A^{2}\right)^{7 / 2}, \quad G_{10}=\frac{G_{11}}{2 \pi R_{\tau}}
$$


In terms of string theory parameters

$$
I_{0 \mathrm{~A} / 0 \mathrm{~B}}=\frac{7^{6} g_{0 A}^{5} R_{y}}{30 \pi^{2} \sqrt{\alpha^{\prime}}}\left(1+a^{2} \frac{R_{y}^{2}}{4 \alpha^{\prime} g_{0 A}^{2}}\right)^{7 / 2} .
$$

Interestingly, for a non-zero $a$ parameter the instanton action has a minimum, non-vanishing value as a function of the coupling $g_{0 A}, I_{0 A}^{\min }=$ const. $R_{y}^{6} a^{5} / \alpha^{\prime 3}$. This means that for $R_{y}^{6} a^{5} \gg \alpha^{\prime 3}$, the action is large and the semiclassical approximation is reliable for any $g_{0 A}$. At small couplings, there is a perturbative instability due to the presence of the type 0 tachyon which dominates over non-perturbative effects. At strong coupling, $g_{0 \mathrm{~A}} \gg 1$, the tachyon is expected to be absent, and the decay should be produced by the formation of the bubble of nothing, with a decay rate given by (4.4). The decay is suppressed at large couplings. This is expected, since at large couplings the dynamics of type $0 \mathrm{~A}$ theory should approach that of M-theory, which is stable (for very large radius $R_{\tau}$, the periodicity of the fermions should not significantly affect the dynamics).

The decay rate of type $0 \mathrm{~A}$ theory at strong coupling when $\tilde{y}$ is non-compact is given by the Witten decay rate of a space $\mathbb{M}^{10} \times S^{1}$. The rate can be obtained from the formulas of the appendix by setting $D=11, A=0$ and replacing $G_{12} / 2 \pi R_{y} \rightarrow G_{11}$. We find

$$
I_{0 \mathrm{~A}}=\frac{\Omega_{9}}{8 G_{11}} 2^{16} R_{\tau}^{9}=\frac{2^{10} \pi^{4} R_{\tau}^{8}}{3 G_{10}}=\frac{2^{15}}{3 \pi^{2}} g_{0 \mathrm{~A}}^{6} .
$$

We see that the action increases with the string coupling to the power six, so the decay rate is rapidly suppressed as the coupling is increased.

\section{Acknowledgments}

We would like to thank R. Emparan and L. Tagliacozzo for useful remarks. This work is partially supported by CYT FPA 2004-04582-C02-01 and CIRITGC 2001SGR-00065. JLC is also supported by the Spanish's Ministerio de Educación y Ciencia, FPU fellowship (ref: AP2003-4193).

\section{A. Decay of $\mathbb{M}^{D-1} \times T^{2}$}

\section{A.1 The $(D+1)$-dimensional instanton}

We can obtain the $(D+1)$-dimensional version of our instanton from the $D$-dimensional charged Kaluza-Klein black hole solution given in [7]. By uplifting the solution to $D+1$ dimensions, and a Wick rotation along with $Q \rightarrow i q$, we find

$$
\begin{aligned}
\mathrm{d} s_{\text {inst. }}^{2}= & \frac{1}{1+\alpha / r^{D-3}}\left(\left(1-\left(r_{h} / r\right)^{D-3}\right)+\frac{2 q^{2}}{(D-3)^{2} \Omega_{D-2}^{2}} \frac{1}{r^{2(D-3)}}\right) \mathrm{d} \tau^{2} \\
& +\frac{\mathrm{d} r^{2}}{1-\left(r_{h} / r\right)^{D-3}}+r^{2} \mathrm{~d} \Omega_{D-2}^{2}+\left(1+\alpha / r^{D-3}\right) \mathrm{d} y^{2} \\
& -\frac{2 \sqrt{2} q}{(D-3) \Omega_{D-2}} \frac{1}{r^{D-3}} \mathrm{~d} y \mathrm{~d} \tau, \quad \Omega_{D-2}=\frac{2 \pi^{\frac{D-1}{2}}}{\Gamma\left(\frac{D-1}{2}\right)}
\end{aligned}
$$


Following the same procedure as in section 2, we consider the Killing vector $\zeta=\partial_{\tau}+A \partial_{y}$, where

$$
A=\frac{\sqrt{2} q}{(D-3) \Omega_{D-2} r_{h}^{D-3}} \frac{1}{1+\alpha / r_{h}^{D-3}},
$$

which has zero norm at the horizon, and introduce the $\tilde{y}=y-A \tau$ variable, which is constant along the orbits of $\zeta$. In order to remove the potential conical singularity, $\tau$ has to be $\left(2 \pi R_{\tau}\right)$-periodic at fixed $\tilde{y}$ coordinate, with $R_{\tau}$ now given by

$$
R_{\tau}=\frac{2}{D-3} r_{h} \sqrt{1+\alpha / r_{h}^{D-3}} .
$$

Again, $A$ represents the value of the gauge field at infinity, $\tilde{A}_{\tau}(\infty)=A$. In order to express the solution in terms of parameters $R_{\tau}$ and $A$, it is first convenient to introduce $k$ defined by

$$
k=1-\sqrt{1-2 \frac{D-2}{(D-3)^{2}}\left(\frac{q}{\kappa^{2} M}\right)^{2}} .
$$

The parameters are then given by

$$
\begin{aligned}
q & =(D-3) \kappa^{2} M \sqrt{\frac{k(2-k)}{2(D-2)}}, \\
r_{h}^{D-3} & =\frac{2 \kappa^{2} M}{(D-2) \Omega_{D-2}}\left(1+\frac{D-3}{2} k\right), \quad \alpha=-\frac{\kappa^{2} M}{\Omega_{D-2}} k, \\
\kappa^{2} M & =\frac{(D-2)(D-3)^{D-3}}{2^{D-2}} \Omega_{D-2} R_{\tau}^{D-3}\left(1+\frac{D-3}{2} k\right)^{(D-5) / 2}\left(1-\frac{k}{2}\right)^{-(D-3) / 2} .
\end{aligned}
$$

The instanton solution is real for $k$ in the range $0 \leq k \leq 2$ (so that $q$ is real). Using

$$
A=\sqrt{\frac{(D-2) k}{2-k}},
$$

i.e.

$$
k=\frac{2 A^{2}}{(D-2)+A^{2}} .
$$

we obtain

$$
\begin{aligned}
q & =\frac{(D-3)^{D-2}}{2^{D-5 / 2}} \Omega_{D-2} R_{\tau}^{D-3} A\left(1+A^{2}\right)^{(D-5) / 2}, \\
r_{h} & =\frac{D-3}{2} R_{\tau} \sqrt{1+A^{2}} \\
\alpha & =-\left(\frac{D-3}{2} R_{\tau}\right)^{D-3} A^{2}\left(1+A^{2}\right)^{(D-5) / 2} .
\end{aligned}
$$

At infinity, the instanton approaches the metric

$$
\mathrm{d} s_{D+1}^{2}=\mathrm{d} y^{2}+\mathrm{d} \tau^{2}+\mathrm{d} r^{2}+r^{2} \mathrm{~d} \Omega_{D-2}^{2}
$$

with the identification

$$
(y, \tau)=\left(y+2 \pi n R_{y}+2 \pi m A R_{\tau}, \tau+2 \pi m R_{\tau}\right) .
$$




\section{A.2 The instanton action}

In $D$ dimensions the solution represents an electrically charged black hole. The thermodynamics is as follows. The Hawking temperature and entropy are

$$
\begin{gathered}
T=\frac{1}{2 \pi R_{\tau}}=\frac{D-3}{4 \pi r_{h} \sqrt{1+\alpha / r_{h}^{D-3}}}, \\
S_{D}=\frac{\Omega_{D-2} r_{h}^{D-2}}{4 G_{D}}\left(1+\alpha / r_{h}^{D-3}\right)^{1 / 2} .
\end{gathered}
$$

The action can be computed as the free energy over the temperature. Using $F=M-$ $T S-\Phi_{H} Q$ and the Smarr formula []]

$$
M=\frac{D-2}{D-3} T S+\Phi_{H} Q
$$

we find

$$
\begin{aligned}
I_{\text {inst. }} & =\frac{F}{T}=\frac{S}{D-3}=2 \pi R_{y} \frac{\Omega_{D-2}}{4 G_{D+1}} \frac{r_{h}^{D-2}}{(D-3)}\left(1+\alpha / r_{h}^{D-3}\right)^{1 / 2} \\
& =\frac{\Omega_{D-2}}{16 \pi G_{D+1}}\left(2 \pi R_{y}\right)\left(2 \pi R_{\tau}\right) r_{h}^{D-3}
\end{aligned}
$$

where $G_{D+1}=2 \pi R_{y} G_{D}$ and we have used (A.11).

The instanton action can also be computed directly from its definition as an integral,

$$
I_{\text {inst. }}=-\frac{1}{16 \pi G_{D+1}} \int \mathrm{d}^{D+1} x \sqrt{g} R-\frac{1}{8 \pi G_{D+1}} \oint \sqrt{h}\left(K-K_{0}\right)
$$

Following the same method as in section 3.1, we obtain

$$
\begin{aligned}
I_{\text {inst. }} & =\frac{1}{16 \pi G_{D+1}} \int \mathrm{d} \Omega_{D-2} A\left(\theta_{i}\right) \int \mathrm{d} \tau \mathrm{d} y r_{h}^{D-3} \\
& =\frac{\Omega_{D-2}}{16 \pi G_{D+1}}\left(2 \pi R_{y}\right)\left(2 \pi R_{\tau}\right) r_{h}^{D-3} .
\end{aligned}
$$

which exactly agrees with (A.14).

The decay rate is thus given by $\Gamma \sim e^{-I_{\text {inst. }}}$, with

$$
I_{\text {inst. }}=I_{0}\left(1+A^{2}\right)^{(D-3) / 2},
$$

where

$$
I_{0}=\frac{\pi \Omega_{D-2}}{4 G_{D+1}}\left(\frac{D-3}{2}\right)^{D-3} R_{y} R_{\tau}^{D-2} .
$$

We have used (A.8b). The qualitative features are as in the decay of the six dimensional space $\mathbb{M}^{4} \times T^{2}$. 


\section{References}

[1] E. Witten, "Instability Of The Kaluza-Klein Vacuum," Nucl. Phys. B 195 (1982) 481.

[2] S. R. Coleman, "The Fate Of The False Vacuum. 1. Semiclassical Theory," Phys. Rev. D 15, 2929 (1977) [Erratum-ibid. D 16, 1248 (1977)].

[3] S. R. Coleman and F. De Luccia, "Gravitational Effects On And Of Vacuum Decay," Phys. Rev. D 21 (1980) 3305.

[4] F. Dowker, J. P. Gauntlett, G. W. Gibbons and G. T. Horowitz, "The Decay of magnetic fields in Kaluza-Klein theory," Phys. Rev. D 52 (1995) 6929 [arXiv:hep-th/9507143].

[5] J. G. Russo and A. A. Tseytlin, "Magnetic flux tube models in superstring theory," Nucl. Phys. B 461, 131 (1996) [arXiv:hep-th/9508068].

[6] G. W. Gibbons and D. L. Wiltshire, "Black Holes In Kaluza-Klein Theory," Annals Phys. 167 (1986) 201 [Erratum-ibid. 176 (1987) 393].

[7] G. W. Gibbons and K. i. Maeda, "Black Holes And Membranes In Higher Dimensional Theories With Dilaton Nucl. Phys. B 298, 741 (1988).

[8] A. Chamblin and R. Emparan, "Bubbles in Kaluza-Klein theories with space- or time-like internal dimensions," Phys. Rev. D 55, 754 (1997) [arXiv:hep-th/9607236].

[9] H. Elvang, T. Harmark and N. A. Obers, "Sequences of bubbles and holes: New phases of Kaluza-Klein black holes," JHEP 0501, 003 (2005) [arXiv:hep-th/0407050].

[10] R. Emparan and H. S. Reall, "Generalized Weyl solutions," Phys. Rev. D 65, 084025 (2002) [arXiv:hep-th/0110258].

[11] O. Bergman and M. R. Gaberdiel, "Dualities of type 0 strings," JHEP 9907, 022 (1999) [arXiv:hep-th/9906055].

[12] L. J. Dixon and J. A. Harvey, "String Theories In Ten-Dimensions Without Space-Time Supersymmetry," Nucl. Phys. B 274, 93 (1986); N. Seiberg and E. Witten, "Spin Structures In String Theory," Nucl. Phys. B 276, 272 (1986).

[13] M. S. Costa and M. Gutperle, "The Kaluza-Klein Melvin solution in M-theory," JHEP 0103, 027 (2001) [arXiv:hep-th/0012072].

[14] J. G. Russo and A. A. Tseytlin, "Magnetic backgrounds and tachyonic instabilities in closed superstring theory and M-theory," Nucl. Phys. B 611, 93 (2001) [arXiv:hep-th/0104238].

[15] R. Emparan and M. Gutperle, "From p-branes to fluxbranes and back," JHEP 0112, 023 (2001) [arXiv:hep-th/0111177]. 